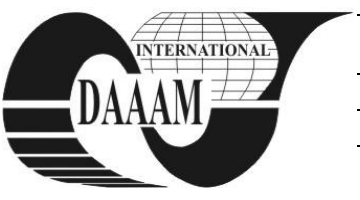

\title{
THE HAMMERSTEIN AND WIENER MODELS IN NONLINEAR PROCESS CONTROL
}

\author{
BABIK, Z[denek] \& DOSTAL, P[etr]
}

Abstract: The application of Hammerstein and Wiener models in controling of nonlinear systems is described in this paper. The pole placement methods are used for designing of timediscrete controllers. Equations which are used for design are presented too. This method has been tested by simulations in program MathWorks MATLAB $2010 b$.

Key words: nonlinear control, Hammerstein and Wiener models, static nonlinearity, nonlinear system

\section{INTRODUCTION}

The control of nonlinear processes is an area of process control theory which is examined much fewer than classical linear control theory. But in practice, the most of real processes have nonlinear behavior and their linearization is possibly only in a near neighborhood of an operating point. These processes can be controlled by linear PID or polynomial controllers with many limitations; and also their control quality is not an optimal.

One solution of this problem which is based on the fact that many real processes can be factorable - they are consisting from nonlinear static parts and linear dynamic parts - is presented in this paper. On the basis of their mutual position Hammerstein or Wiener model can be distinguished.

\section{HAMMERSTEIN AND WIENER MODELS}

In Fig. 1 and Fig. 2, we can see a basic Hammerstein and Wiener models.



Fig. 1. Hammerstein model of nonlinear system

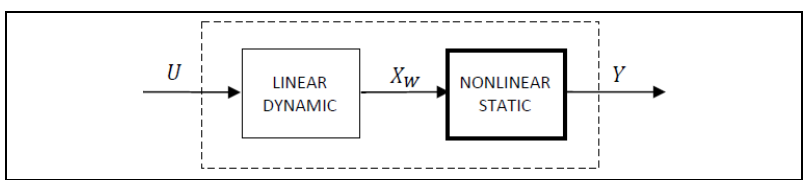

Fig. 2. Wiener model of nonlinear system

The basic Hammerstein model $\boldsymbol{N}_{\boldsymbol{H}}$ is a cascade structure of nonlinear static block and linear dynamic block which can be described by next formulas:

$$
\begin{gathered}
X_{H}(k)=f_{H}(U(k)) \\
Y(k)=b_{0} X_{H}(k)+b_{1} X_{H}(k-1)+\cdots+b_{m} X_{H}(k-m) \\
-a_{1} Y(k-1)-\cdots-a_{n} Y(k-n)
\end{gathered}
$$

The basic Wiener model $N_{W}$ is a cascade structure of the linear dynamic block and nonlinear static block which can be described by next formulas:

$$
\begin{aligned}
X_{W}(k)=b_{0} U(k)+ & b_{1} U(k-1)+\cdots+b_{m} U(k-m) \\
& -a_{1} X_{W}(k-1)-\cdots-a_{n} X_{W}(k-n)
\end{aligned}
$$

$$
Y(k)=f_{W}\left(X_{W}(k)\right)
$$

The H-models can describe many different processes, especially if their main nonlinear behavior is caused by actuators (dead zone, saturation, etc.).

The W-models are appropriate for systems whose outputs are measured by sensors with nonlinear characteristic or for a controller design.

\section{BASIC PROCEDURE OF DESIGN}

In Fig. 3, we can see a schematic illustration of the basic procedure which can be used for linearizing of non-linear processes.

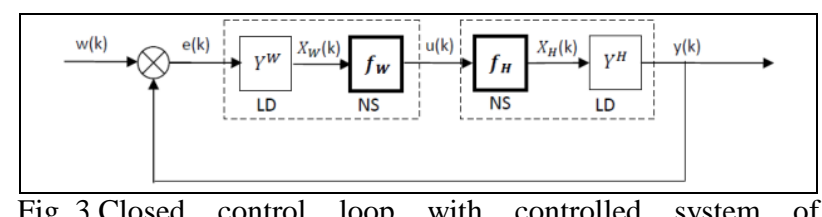

Hammerstein type and controller of Wiener type.

$$
\begin{aligned}
& \text { If we choose } \boldsymbol{f}_{\boldsymbol{W}}=\boldsymbol{f}_{\boldsymbol{H}}^{-\mathbf{1}} \text { then we can write (from Fig. 3): } \\
& \begin{aligned}
X_{H}(k)=\boldsymbol{f}_{\boldsymbol{H}}(u(k))=\boldsymbol{f}_{\boldsymbol{H}}\left(\boldsymbol{f}_{\boldsymbol{W}}\left(X_{W}(k)\right)\right. \\
=\boldsymbol{f}_{\boldsymbol{H}}\left(\boldsymbol{f}_{\boldsymbol{H}}^{-1}\left(X_{W}(k)\right)\right)=X_{W}(k)
\end{aligned}
\end{aligned}
$$

From formula (5) we can make the following finish: If we can describe the nonlinearity and if it can be inversible (minimally partly) then we have to study only the properties of the linear dynamic of controlled system for a design of controllers.

\section{DESIGN OF CONTROLLER}

The linear dynamic part will be assumed in form

$$
G(s)=\frac{K}{\left(T_{1} s+1\right)\left(T_{2} s+1\right)}
$$

where: $K$ - gain, $T_{1}, T_{2}$ - time constants. This transfer function will be transformed into the second order discrete system:

$$
G(z)=\frac{Y(z)}{U(z)}=\frac{B\left(z^{-1}\right)}{A\left(z^{-1}\right)}=\frac{b_{1} z^{-1}+b_{2} z^{-2}}{1+a_{1} z^{-1}+a_{2} z^{-2}}
$$

Transfer function of controller will be assumed in form:

$$
G_{R}(z)=\frac{U(z)}{E(z)}=\frac{Q\left(z^{-1}\right)}{P\left(z^{-1}\right)}=\frac{q_{0}+q_{1} z^{-1}+q_{2} z^{-2}}{\left(1-z^{-1}\right)\left(1+\gamma z^{-1}\right)}
$$

From (8), we can establish the difference formula for computation of a control action:

$$
\begin{aligned}
u(k)=q_{0} e(k)+q_{1} e(k-1)+q_{2} e(k-2) & \\
& +(1-\gamma) u(k-1)+\gamma u(k-2)
\end{aligned}
$$

For the control transfer function of the closed control loop we can write:

$$
G_{W / Y}(z)=\frac{Y(z)}{W(z)}=\frac{B\left(z^{-1}\right) Q\left(z^{-1}\right)}{A\left(z^{-1}\right) P\left(z^{-1}\right)+B\left(z^{-1}\right) Q\left(z^{-1}\right)}
$$

The denominator of (10) is called as a characteristic polynomial. By the choice of the characteristic polynomial: 


$$
G_{W / Y}(z)=\frac{Y(z)}{W(z)}=\frac{B\left(z^{-1}\right) Q\left(z^{-1}\right)}{A\left(z^{-1}\right) P\left(z^{-1}\right)+B\left(z^{-1}\right) Q\left(z^{-1}\right)}
$$

in the condition polynomial formula:

$$
A\left(z^{-1}\right) P\left(z^{-1}\right)+B\left(z^{-1}\right) Q\left(z^{-1}\right)=D\left(z^{-1}\right)
$$

we establish the poles of transfer function (10). We can affect the control quality by the pole placement.

\section{STATIC NONLINEARITY LINEARIZATION}

AND

ITS

The static nonlinearity of controlled system we will be assumed in form (look at the Fig. 4):

$$
\begin{aligned}
& u \geq \boldsymbol{u}_{1 \text { max }} \\
& y=y_{\max } \\
& \boldsymbol{u}_{1 \text { min }} \leq \boldsymbol{u} \leq \boldsymbol{u}_{1 \text { max }} \quad y^{+}=\frac{y_{\max }}{u_{1 \max }-u_{1 \min }} \\
& \left(u-u_{1 \min }\right)=K^{+} \cdot\left(u-u_{1 \min }\right) \\
& \boldsymbol{u}_{2 \text { min }} \leq \boldsymbol{u} \leq \boldsymbol{u}_{1 \text { mín }} \quad y=0 \\
& \boldsymbol{u}_{2 \max } \leq \boldsymbol{u} \leq \boldsymbol{u}_{2 \min } \quad y^{-}=\frac{y_{\min }}{u_{2 \max }-u_{2 \min }} \\
& \boldsymbol{u} \leq \boldsymbol{u}_{1 \min } \\
& \left(u-u_{2 \min }\right)=K^{-} \cdot\left(u-u_{1 \min }\right)
\end{aligned}
$$

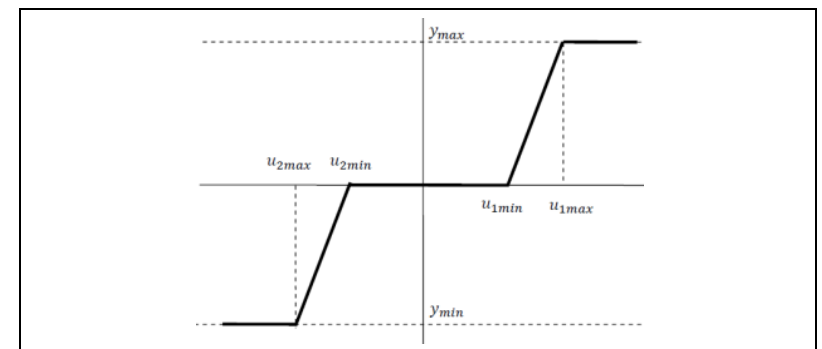

Fig. 4. Static non-linearity

For the best linearization of nonlinearity (13) we must consider the following manner:

- The saturation of the control action we cannot compensate but we should include it into the computation of the control action.

- The dead zone of the control action we can compensate in the following way:

$$
\begin{array}{ll}
\left\langle\mathbf{0}, \boldsymbol{u}_{1 \max }\right\rangle: & u(k)=\boldsymbol{f}_{\boldsymbol{W}}\left(X_{w}(k)\right)=X_{w}(k)+u_{1 \min } \\
\left\langle\boldsymbol{u}_{2 \max }, \mathbf{0}\right\rangle: & u(k)=\boldsymbol{f}_{\boldsymbol{W}}\left(X_{w}(k)\right)=X_{w}(k)+u_{2 \min }
\end{array}
$$

For the control action computation had to be used the values of $X_{w}(k)$, we cannot use the linearized values of $u(k)$.

\section{MEASUREMENT}

Controlled-system and controller parameters:

The time-continuous transfer function:

$$
G(s)=\frac{K}{\left(T_{1} s+1\right)\left(T_{2} s+1\right)}=\frac{1}{(5 s+1)(2 s+1)}
$$

The discrete transfer function for the sampling period $T_{0}=2.5 \mathrm{~s}$ :

$$
G\left(z^{-1}\right)=\frac{0.1801 z^{-1}+0.1006 z^{-2}}{1-0.893 z^{-1}+0.1738 z^{-2}}
$$

The non-linearity parameters.

$$
u_{1 \min }=1, u_{1 \max }=5, u_{2 \min }=-1.5, u_{2 \max }=-5 \text {, }
$$$$
y_{\max }=5, y_{\min }=-5
$$

The characteristic polynomial $\left(z^{-1}\right)$ :

$$
D\left(z^{-1}\right)=1+d_{1} z^{-1}+d_{2} z^{-2}
$$

where:

$$
\begin{gathered}
d_{1}=-2 \cdot e^{-\xi \omega_{n} T_{0}} \cdot \cos \left(\omega_{n} T_{0} \sqrt{1-\xi^{2}}\right) \text { for } \xi \leq 1 \\
d_{1}=-2 \cdot e^{-\xi \omega_{n} T_{0}} \cdot \cosh \left(\omega_{n} T_{0} \sqrt{1-\xi^{2}}\right) \text { for } \xi>1 \\
d_{2}=e^{-2 \xi \omega_{n} T_{0}}
\end{gathered}
$$

where: $\omega_{n}$ - circular frequency, $\xi$ - damping ratio

The transfer function of controller:

$$
G\left(z^{-1}\right)=\frac{0.7800-0.9424 z^{-1}+0.3367 z^{-2}}{\left(1-z^{-1}\right)\left(1+0.1949 z^{-1}\right)}
$$

Control process:

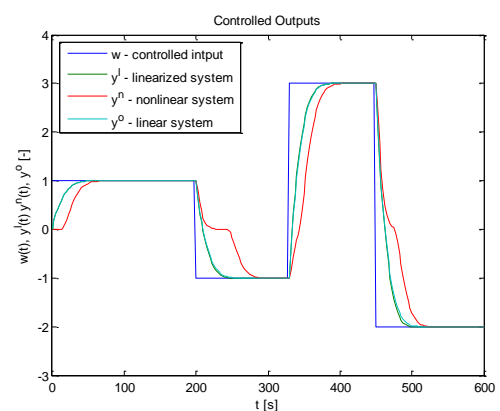

Fig. 5. Controlled outputs

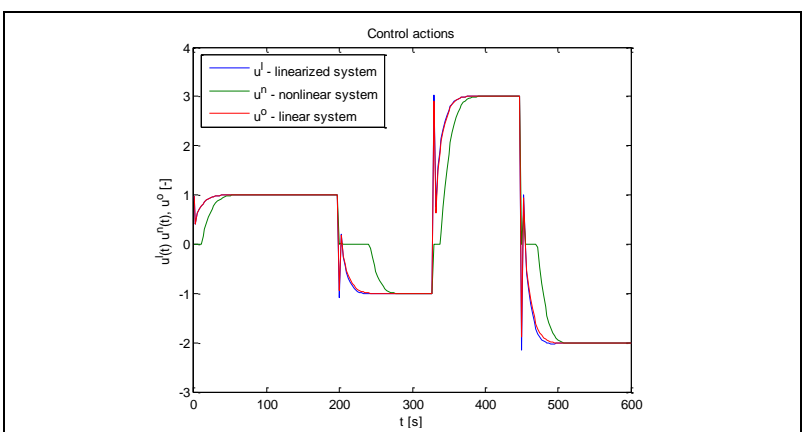

Fig. 6. Control actions

\section{DISCUSSSION}

The following truth could be said from the measured data which are displayed in Fig. 5 and Fig. 6: The process control quality was manifold better when the linearizing discrete feedback controller was used, than the classical discrete feedback controller was used only.

On the other hand, there exist some restrictions: This method can be used only for the factorable systems and the basic version of this method is non-universal - for each process must be designed the special nonlinear feedback controller.

The universality of this method must be increased by future research. One possible way is using of methods from genetic programming field or from neural network field.

\section{ACKNOWLEDGEMENTS}

This work was partly supported by the Ministry of Education of the Czech Republic under the grant MSM 7088352101 and by TBU in Zlin under the grant IGA/55/FAI/10/D.

\section{REFERENCES}

Nelles, O. (2001). Nonlinear System Identification, 1. release, Heidelberg: Springer-Verlag, ISBN 3-540-67369-5, Berlin

Corriou, J.-P. (2004). Process control Theory and Applications, 1. release, London: Springer-Verlag, ISBN 1-85233-776-1, London

Balátě, J. (2003). Automatic control, 1. release, BEN - technical literature, ISBN 80-7300-020-2, Praque

Bobál, V. (2008). Adaptive and predictive control, 1. release, Thomas Bata University, Faculty of Applied Informatic, ISBN 80-7318-662-3, Zlín

Bányász, Cs., Keviczky L. (2002). A simple PID regulator applicable for a class of factorable nonlinear plants, In: Proceedings of the American Control Conference Anchorage, 2354-2359, ISBN 0-7803-7298-0 